\title{
Association of Incidence between Pancreatic Adipose Infiltration and Metabolic Syndrome: A Literature Review and Meta-analysis
}

\author{
Ying Zhang $(\mathbb{D}$, Jing $\mathrm{Li}(\mathbb{D}$, and Youqing Xu $\mathbb{C}$ \\ Department of Gastroenterology, Beijing Tiantan Hospital, Capital Medical University, Fengtai District, Beijing 100070, China \\ Correspondence should be addressed to Youqing Xu; dryouqingxu@163.com
}

Received 4 August 2021; Revised 23 September 2021; Accepted 29 September 2021; Published 5 November 2021

Academic Editor: Tao Huang

Copyright (C) 2021 Ying Zhang et al. This is an open access article distributed under the Creative Commons Attribution License, which permits unrestricted use, distribution, and reproduction in any medium, provided the original work is properly cited.

\begin{abstract}
Objective. To investigate association of incidence between pancreatic adipose infiltration and metabolic syndrome (METS). Methods. We searched PubMed, Embase, Cochrane Library, and Web of Science databases up to July 2021. We compared incidence rate of METS between adults with and without pancreatic adipose infiltration, along with their clinical features, such as fasting blood glucose (FBG), triglyceride (TG), high-density lipoprotein (HDL), and hypertension (HTN). Cross-sectional study, cohort study, and case control study were included. Two investigators independently completed study selection, data extraction, and risk of bias assessment. Results. Eleven eligible studies that involved 17,127 patients were included, including 8 cross-sectional studies, 2 cohort studies, and 1 case control study. There was a trend of increasing in incidence rate of METS $(\mathrm{OR}=2.66,95 \% \mathrm{CI}: 1.89-3.75)$ of adults with pancreatic adipose infiltration when compared to those without the disease. There was a trend of increasing in $\mathrm{HTN}(\mathrm{OR}=1.68,95 \% \mathrm{CI}: 1.32-2.13)$ and levels of FBG (SMD $=0.54,95 \%$ CI: 0.35-0.72) and TG (SMD $=0.39,95 \% \mathrm{CI}: 0.25-0.53)$ of adults with pancreatic adipose infiltration, while there was a trend of decreasing in HDL level (SMD $=-0.29,95 \%$ CI: $-0.43 \sim-0.15)$. Conclusion. There was an association of incidence between pancreatic adipose infiltration and METS. Indicators of clinical features related to pancreatic adipose infiltration were more likely to arise, such as FBG and TG levels and HTN, but HDL level tended to decrease.
\end{abstract}

\section{Introduction}

With higher standards of living, metabolic syndrome (METS) becomes a global health issue attributable to eating high-fat and high-calorie foods and lacking exercise in all age and socioeconomic groups $[1,2]$. METS refers to pathological state of metabolic disorder in protein, adipose, and carbohydrate, which is a set of complex disorders containing cardiometabolic syndrome, insulin resistance syndrome, etc. $[3,4]$. Obesity, diabetes mellitus (DM), hypertension, dyslipidemia, high blood viscosity, and high uric acid levels may be one of the components of METS.

Ectopic fat can accumulate in organs such as the liver, muscle, heart, and pancreas, which is an essential pathophysiological abnormality of METS [5, 6]. Nonalcoholic fatty liver disease (NAFLD) is an example of ectopic fat accumulation in the liver. From simple steatosis, the disease may progress to nonalcoholic steatohepatitis (NASH) to hepatic cirrhosis and hepatic failure [7]. A number of studies suggest an association between NAFLD and METS [8-10]. The incidence rate of NAFLD is increased conspicuously in patients with DM concomitant obesity, while that ranges $40 \%-70 \%$ in DM patients [11] and 57\%-98\% in overweight and obese individuals [8]. Besides, a study [12] manifested an association between adipose infiltration of the liver and metabolic rate.

Few studies focused on the impact and clinical significance of ectopic fat accumulation in pancreas when compared with NAFLD in the past. But it has become the forefront of academic research recently [13]. The pancreas is a retroperitoneal organ that is hard for noninvasive investigations [14]. A high echogenicity of the pancreas is presented in routine transabdominal ultrasound examination. Advanced imaging technologies, such as computer tomography $(\mathrm{CT})$, magnetic resonance imaging (MRI), and high-resolution proton magnetic resonance spectroscopy (1H-MRS), make it possible to evaluate and quantify pancreatic fat noninvasively and accurately $[15,16]$. 


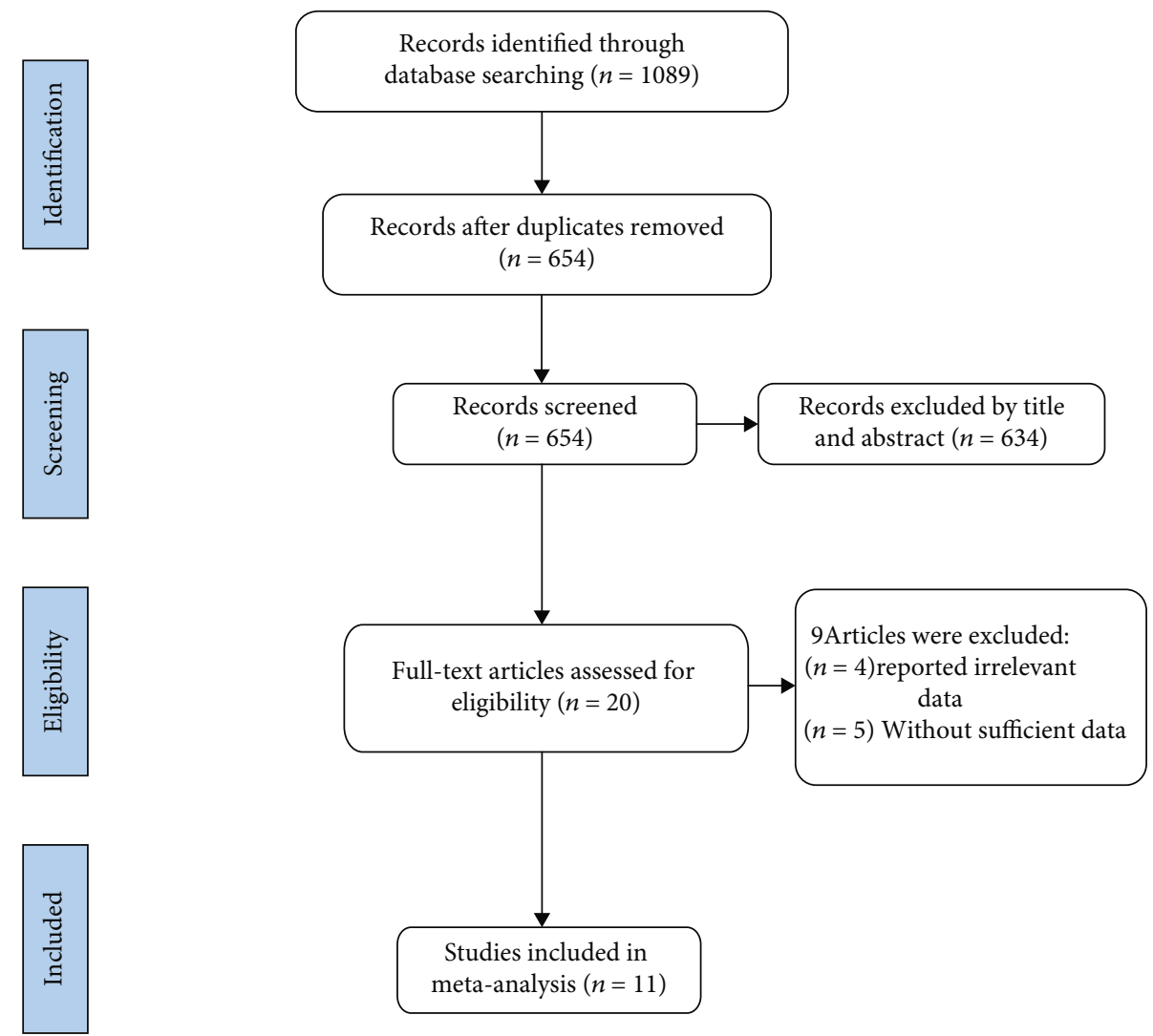

Figure 1: Flow chart of study selection.

Pancreatic adipose infiltration is prevalent and is associated with at least a twofold increase in risks of type 2 diabetes mellitus (T2DM), METS, and hypertension [17, 18]. It is a risk factor for pancreatic cancer and precancerous pancreas as well $[19,20]$. There seems to be some evidence to demonstrate that METS is related to pancreatic adipose infiltration [21, 22]. However, few investigators have drawn on any systematic research into the correlation between pancreatic adipose infiltration and METS [16]. Whether pancreatic adipose infiltration is caused by METS and obesity or a contributing factor to progression of METS would be a fruitful area for further work.

To sum up, we systematically reviewed and compared incidence of METS in adults with and without pancreatic adipose infiltration. We attempted to investigate correlation between pancreatic adipose infiltration and METS and to provide reference for clinical application of routine pancreatic adipose infiltration screening.

\section{Methods}

2.1. Literature Retrieval. This research was strictly followed Preferred Reporting Items for Systematic reviews and Meta-Analyses (PRISMA) statement [23]. PubMed, Embase, Cochrane Library, and Web of Science databases were searched to find relevant articles from inception to July 2021. Search items included "Pancreas," "fatty," "adipose," and "Metabolic Syndrome," etc. The specific strategy for literature retrieval was as follows: ((((Pancreas[MeSH Terms])
OR (Pancreas[Title/Abstract])) OR (pancreatic[Title/Abstract] $))$ AND ((( fatty[Title/Abstract]) OR (adipose[Title/Abstract])) OR (steatosis[Title/Abstract])) OR (Fat[Title/ Abstract $])))$ AND $\quad((((()(($ Metabolic Syndrome $*[\mathrm{MeSH}$ Terms]) OR (Metabolic Syndrome X[MeSH Terms])) OR (Metabolic X Syndrome[MeSH Terms])) OR (Insulin Resistance Syndrome X[MeSH Terms])) OR (Dysmetabolic Syndrome X[MeSH Terms])) OR (Reaven Syndrome X[MeSH Terms])) OR (Metabolic Cardiovascular Syndrome[MeSH Terms])) OR (Cardiometabolic Syndrome*[MeSH Terms])) OR $((((((($ Metabolic Syndrome* [Title/Abstract]) OR (Metabolic Syndrome X[Title/Abstract])) OR (Metabolic X Syndrome[Title/Abstract])) OR (Insulin Resistance Syndrome X[Title/Abstract])) OR (Dysmetabolic Syndrome X[Title/Abstract])) OR (Reaven Syndrome X[Title/Abstract])) OR (Metabolic Cardiovascular Syndrome[Title/Abstract])) OR (Cardiometabolic Syndrome*[Title/Abstract]))). Literature retrieval was completed independently by two investigators. Any discrepancies were resolved by a third investigator.

2.2. Study Selection. Study selection was completed independently by two investigators. Any discrepancies were resolved by a third investigator. Studies included met the following criteria: (1) diagnostic information about pancreatic adipose infiltration and METS and its components was provided; (2) a preliminary report on comparison of incidence rate of METS between adults with and without pancreatic adipose infiltration; (3) at least several results of METS, triglyceride (TG), fasting blood glucose (FBG), high-density lipoprotein 


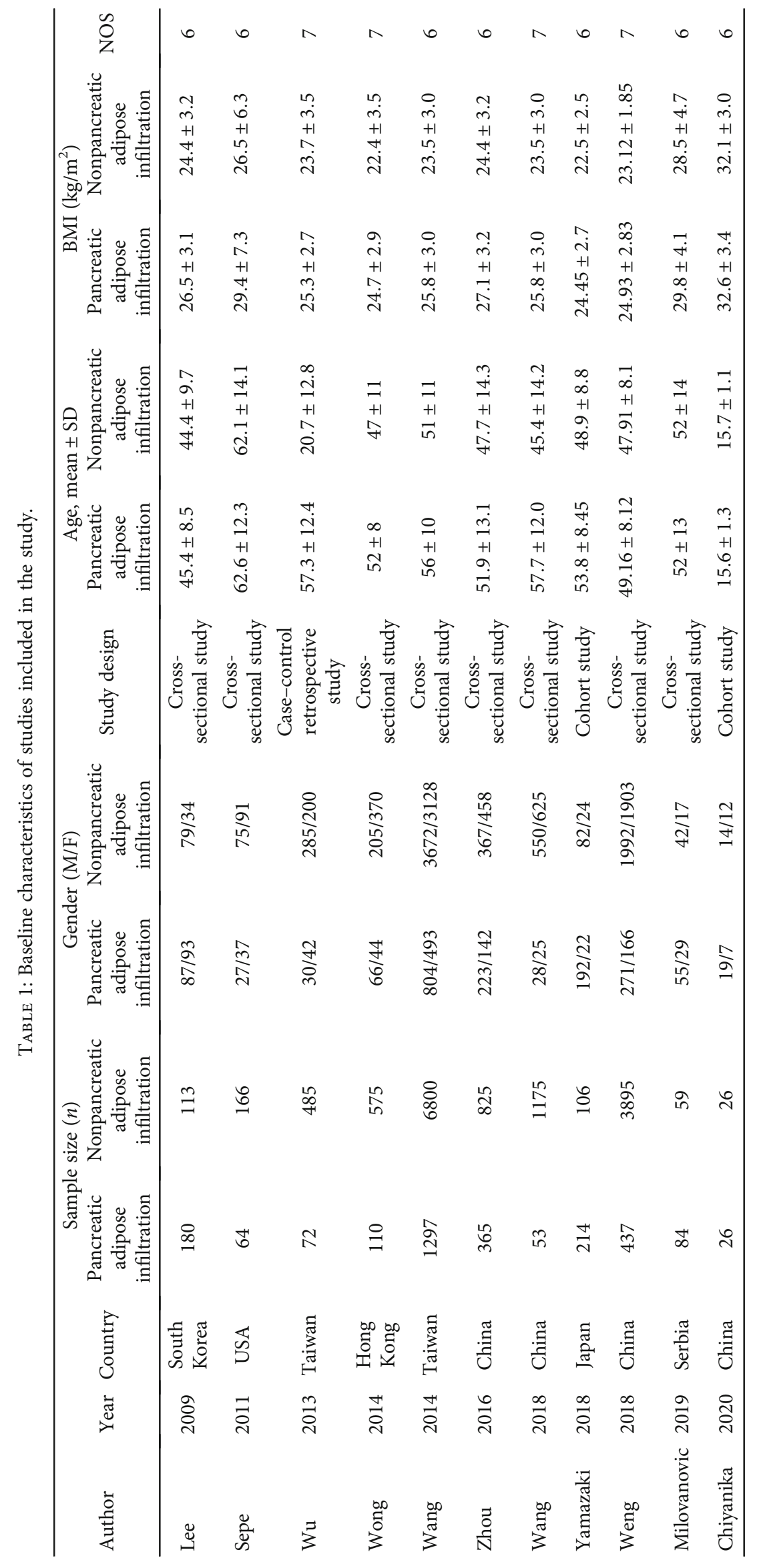




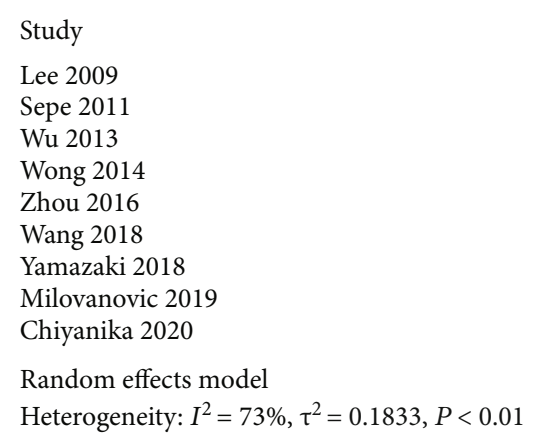

Study

Omitting Lee 2009

Omitting Sepe 2011

Omitting Wu 2013

Omitting Wrong 2014

Omitting Zhou 2016

Omitting Wang 2018

Omitting Yamazaki 2018

Omitting Milovanovic 2019

Omitting Chiyanika 2020

Random effects model

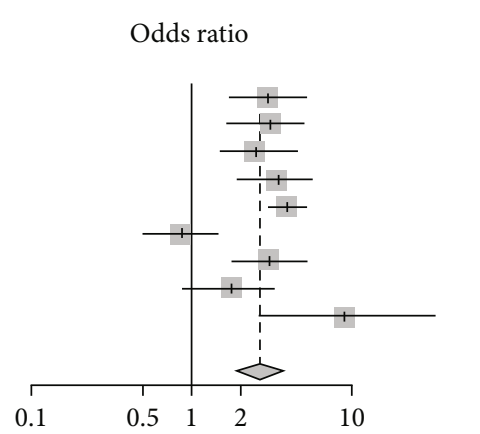

$\begin{array}{rrr}\text { OR } & 95 \%-C I & \text { Weight } \\ 2.97 & {[1.73 ; 5.08]} & 11.8 \% \\ 3.12 & {[1.45 ; 6.72]} & 9.1 \% \\ 2.47 & {[1.44 ; 4.23]} & 11.8 \% \\ 3.33 & {[2.13 ; 5.20]} & 13.0 \% \\ 3.83 & {[2.94 ; 5.00]} & 15.1 \% \\ 0.85 & {[0.49 ; 1.47]} & 11.6 \% \\ 2.93 & {[1.79 ; 4.78]} & 12.4 \% \\ 1.74 & {[0.88 ; 3.43]} & 10.1 \% \\ 9.05 & {[2.57 ; 31.84]} & 5.1 \% \\ 2.66 & {[1.89 ; 3.75]} & 100.0 \%\end{array}$

(a)

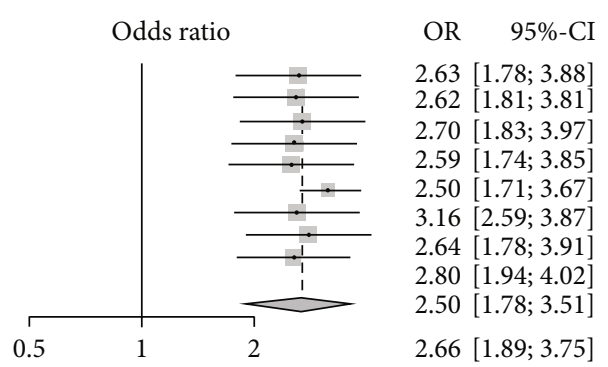

(b)

FIgURE 2: Forest plots of comparison in incidence rate of METS: (a) forest plot comparing the incidence rate of METS; (b) forest plot of prevalence of incidence rate of METS after sensitivity analysis.

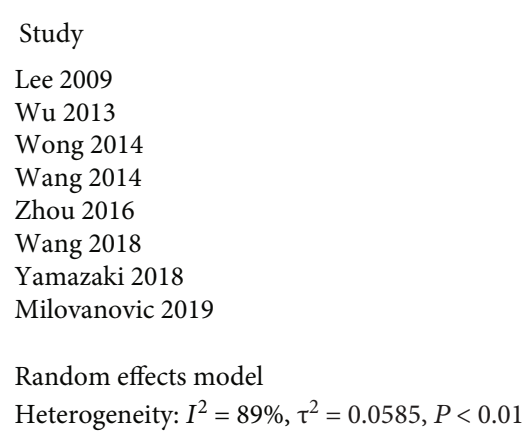

Study

Omitting Lee 2009

Omitting Wu 2013

Omitting Wong 2014

Omitting Wang 2014

Omitting Zhou 2016

Omitting Wang 2018

Omitting Yamazaki 2018

Omitting Milovanovic 2019

Random effects model

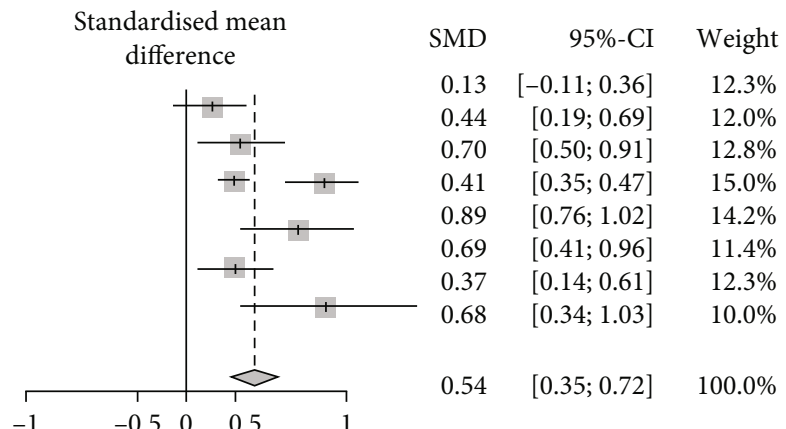

(a)

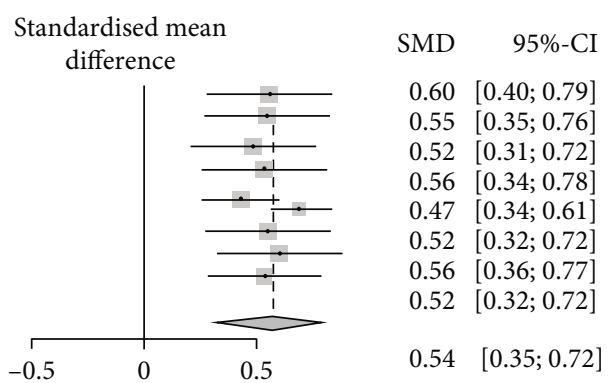

(b)

FIGURE 3: Forest plots of comparison in FBG: (a) forest plot of FBG; (b) forest plot of FBG after sensitivity analysis.

(HDL), and hypertension (HTN) were reported; and (4) the study was a cross-sectional study, cohort study, or casecontrol study. Exclusion criteria were (1) duplicate publications, case series, case reports, reviews, conference abstracts, reviews, editorials, and letters and (2) lack of indicators required for this study or irrelevant data.
2.3. Data Extraction and Quality Assessment. Data extraction and quality assessment were completed independently by two investigators. Any discrepancies were resolved by the third investigator. Information that was isolated from literature included author information, publication year, country, study design, sample size. Patient features contained age, 


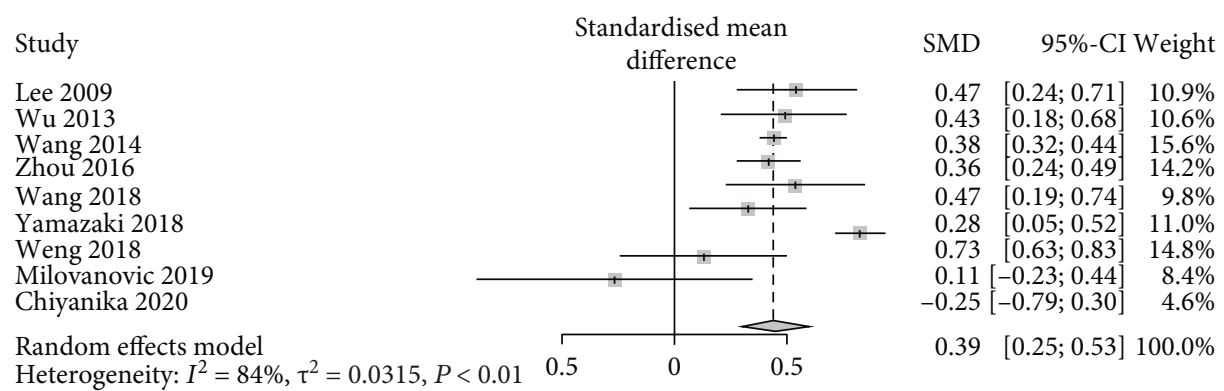

(a)

Study

Omitting Lee 2009

Omitting Wu 2013

Omitting Wang 2014

Omitting Zhou 2016

Omitting Wang 2018

Omitting Yamazaki 2018

Omitting Weng 2018

Omitting Milovanovic 2019

Omitting Chiyanika 2020

Random effects model

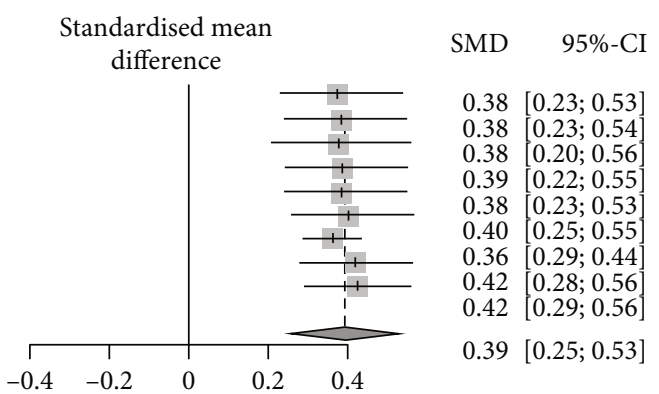

(b)

Figure 4: Forest plots of comparison in TG: (a) forest plot of TG; (b) forest plot of TG after sensitivity analysis.

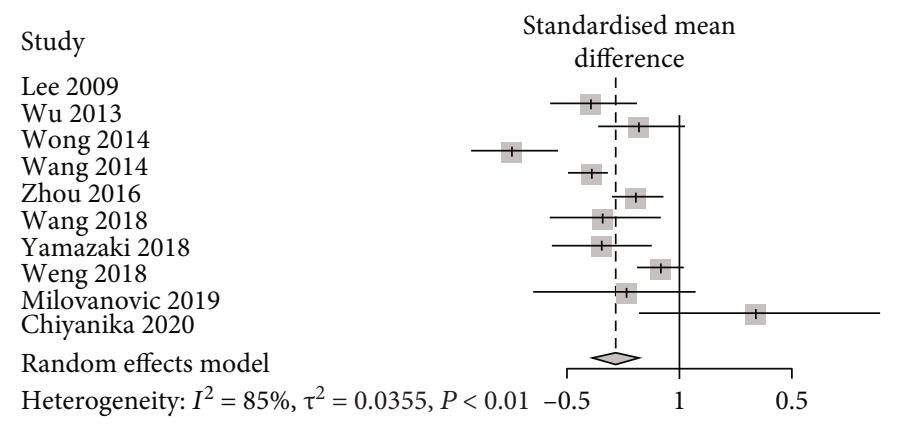

(a)

Study

Omitting Lee 2009

Omitting Wu 2013

Omitting Wong 2014

Omitting Wang 2014

Omitting Zhou 2016

Omitting Wang 2018

Omitting Yamazaki 2018

Omitting Weng 2018

Omitting Milovanovic 2019

Omitting Chiyanika 2020

Random effects model

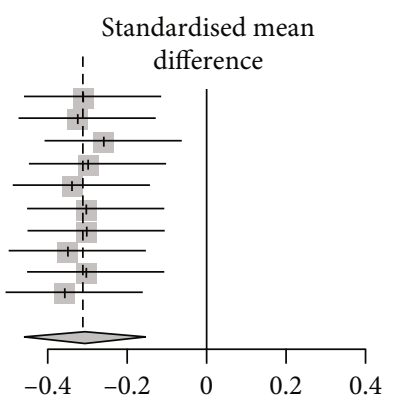

(b) $\begin{array}{lr}\text { SMD } & 95 \%-C I \\ -0.28 & {[-0.43 ;-0.13]} \\ -0.30 & {[-0.45 ;-0.16]} \\ -0.24 & {[-0.37 ;-0.11]} \\ -0.27 & {[-0.44 ;-0.11]} \\ -0.30 & {[-0.46 ;-0.15]} \\ -0.29 & {[-0.44 ;-0.14]} \\ -0.28 & {[-0.43 ;-0.13]} \\ -0.33 & {[-0.46 ;-0.20]} \\ -0.30 & {[-0.44 ;-0.15]} \\ -0.32 & {[-0.46 ;-0.19]} \\ -0.29 & {[-0.43 ;-0.15]}\end{array}$

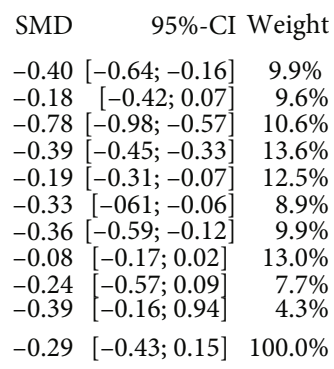

Figure 5: Forest plots of comparison in HDL: (a) forest plot of HDL; (b) forest plot of HDL after sensitivity analysis.

BMI, waist circumference, and incidence rate of pancreatic adipose infiltration. The primary endpoint was incidence rate of METS. Secondary endpoints were FBG, TG, HDL, and HTN.

The Newcastle-Ottawa Quality Assessment Scale (NOS) was used to generate a quality assessment of observational study. It consisted of selection (4 stars), comparability (2 stars), and outcome (3 stars). The total score was 9 stars.
We found that a NOS score of 6 or more can be considered a good quality study. Any discrepancies between the two investigators were resolved by a majority opinion after all items involved were evaluated by the third investigator.

2.4. Statistics. Meta-analysis was completed by the Stata software 16.0. Consecutive data were presented as mean and standard deviation (SD). The specific efficacy was estimated 


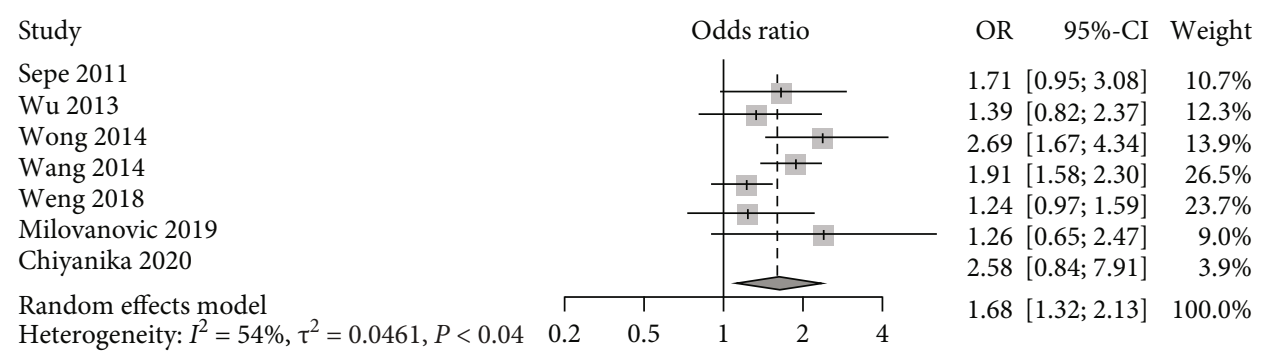

(a)

Study
Omitting Sepe 2011
Omitting Wu 2013
Omitting Wong 2014
Omitting Wang 2014
Omitting Weng 2018
Omitting Milovanovic 2019
Omitting Chiyanika 2020
Random effects model

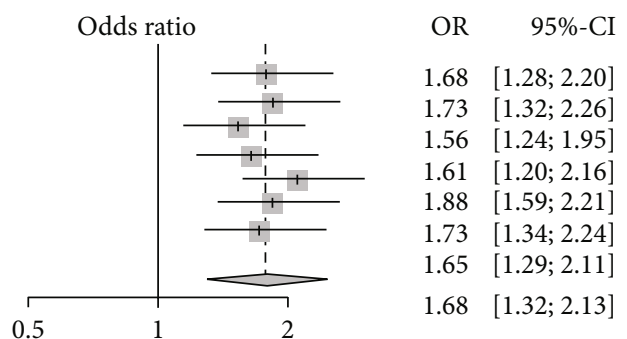

(b)

FIGURE 6: Forest plots of comparison in hypertension: (a) forest plot of hypertension; (b) forest plot of hypertension after sensitivity analysis.

by standardized mean difference (SMD) of consecutive outcomes and 95\% confidence intervals (CIs). For binary outcomes, the combined estimation was the odds ratio (OR) and its $95 \%$ CI. The $I^{2}$ statistic was used to assess heterogeneity of the included studies. Studies with $p<0.1$ or $I^{2}>50 \%$ were considered to have significant heterogeneity, and therefore, a random-effects model was used. Otherwise, a fixedeffects model was initially employed in the analysis. Funnel plots were plotted to assess publication bias in outcome indicators.

\section{Results}

3.1. Literature Retrieval and Study Selection. A total of 1089 records were identified following the established retrieval strategy, and 435 duplicate records were excluded. Then, 634 reports were excluded by initial title and abstract screening, and 20 reports were reviewed in full text. Among them, 4 articles reported irrelevant data, and 5 articles lacked indicators required for this study. Finally, 11 studies were deemed eligible for inclusion [14, 22, 24-32]. Flow chart of study selection was plotted as Figure 1.

3.2. Study Characteristics and Quality Assessment. A total of 11 studies were deemed eligible for inclusion with 2,902 patients with pancreatic adipose infiltration and 14,225 participants without pancreatic adipose infiltration enrolled. 8 cross-sectional studies, 2 cohort studies, and 1 case control study were included. Characteristics and quality assessment of studies included are listed in Table 1. All of these studies were good quality studies with a NOS score of 6 or more.

\subsection{Meta-analysis}

3.3.1. Incidence Rate of METS. 9 studies were analyzed regarding the incidence rate of METS. The results presented a relatively high statistical heterogeneity $\left(I^{2}=73 \%, p<0.01\right)$, and thus, a random-effects model was employed (Figure 2(a)). Taken together, there was a trend of increasing in incidence rate of METS (OR $=2.66,95 \% \mathrm{CI}$ : $1.89-3.75)$ of adults with pancreatic adipose infiltration when compared to those without the disease.

Sensitivity analysis was carried out to investigate potential sources of heterogeneity. Each item was assumed as a potential bias factor and source of heterogeneity. Then, sensitivity analysis was conducted, and forest plots were generated (Figure 2(b)). The results exhibited high heterogeneity.

3.3.2. Secondary Endpoints. Some indicators are bound up with pancreatic adipose infiltration, including FBG, TG, HDL, and HTN.

8 studies compared FBG between patients with and without pancreatic adipose infiltration. The results presented a relatively high statistical heterogeneity $\left(I^{2}=89 \%\right.$, $p<0.01)$, and thus, a random-effects model was employed (Figure 3(a)). Each item was assumed as a potential bias factor and source of heterogeneity. Then, sensitivity analysis was conducted, and forest plots were generated (Figure 3(b)). The results exhibited high heterogeneity. Together, these results illustrated that there was a trend of increase in FBG $(\mathrm{SMD}=0.54,95 \% \mathrm{CI}: 0.35-0.72)$ of adults with pancreatic adipose infiltration.

9 studies compared TG between patients with and without pancreatic adipose infiltration. The results presented a relatively high statistical heterogeneity $\left(I^{2}=84 \%\right.$, $p<0.01)$, and thus, a random-effects model was employed (Figure 4(a)). Each item was assumed as a potential bias factor and source of heterogeneity. Then, sensitivity analysis was conducted, and forest plots were generated (Figure 4(b)). The results exhibited high heterogeneity. Taken together, there was a trend of increase in TG $(\mathrm{SMD}=0.39,95 \% \mathrm{CI}: 0.25-0.53)$ of adults with pancreatic adipose infiltration. 


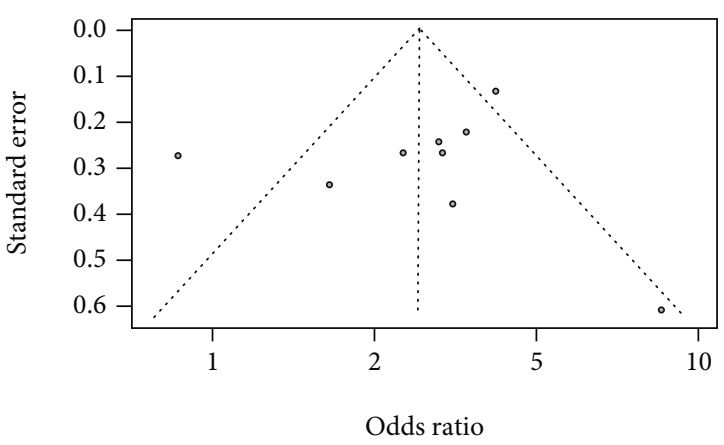

(a)

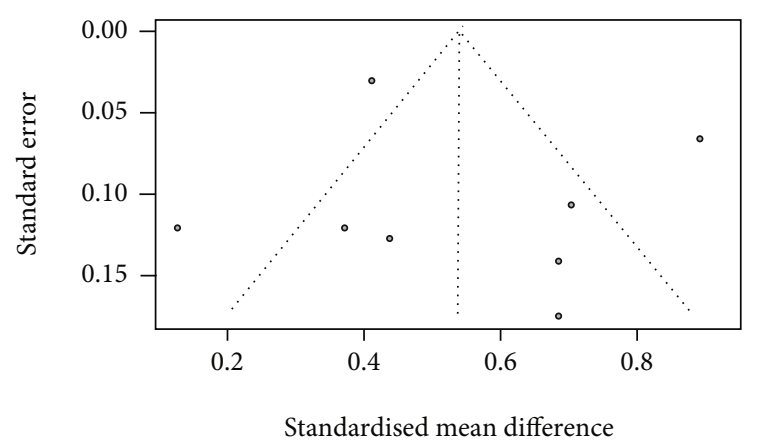

(b)

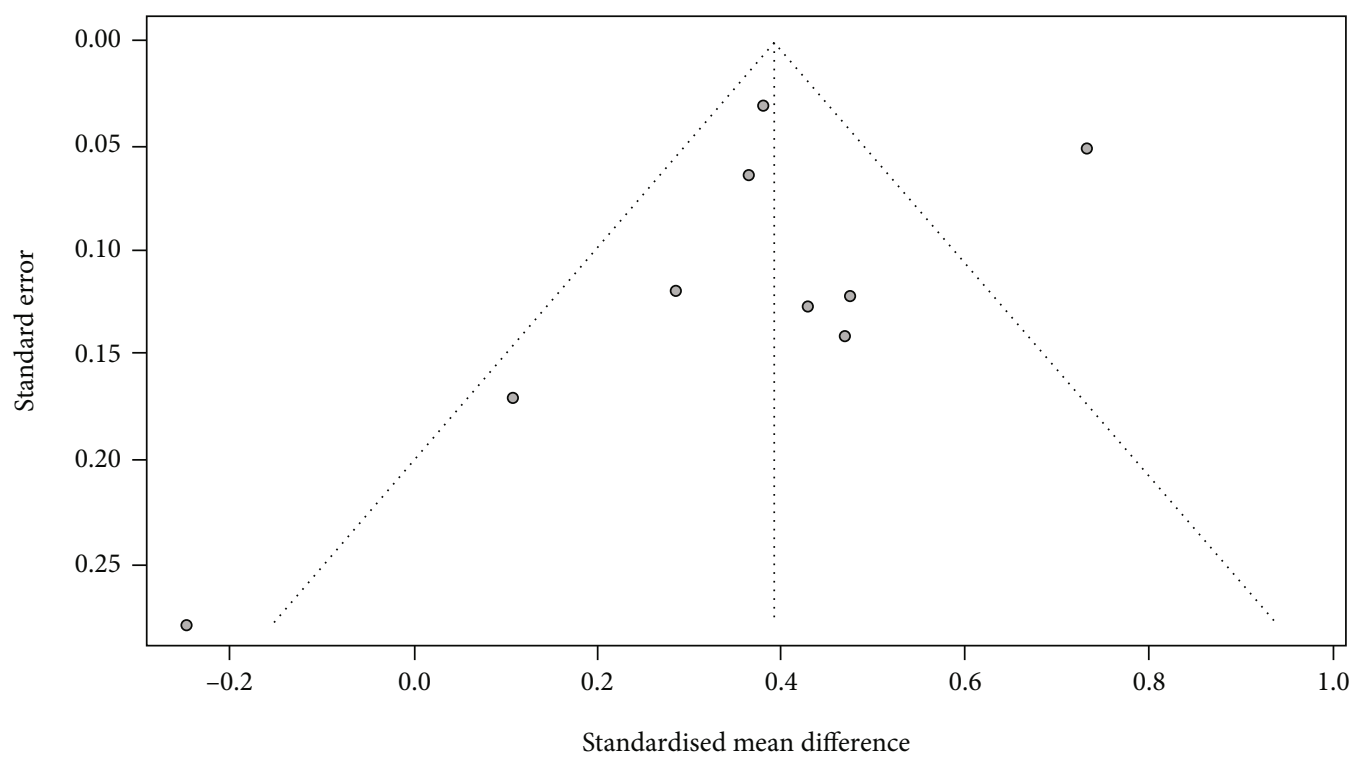

(c)

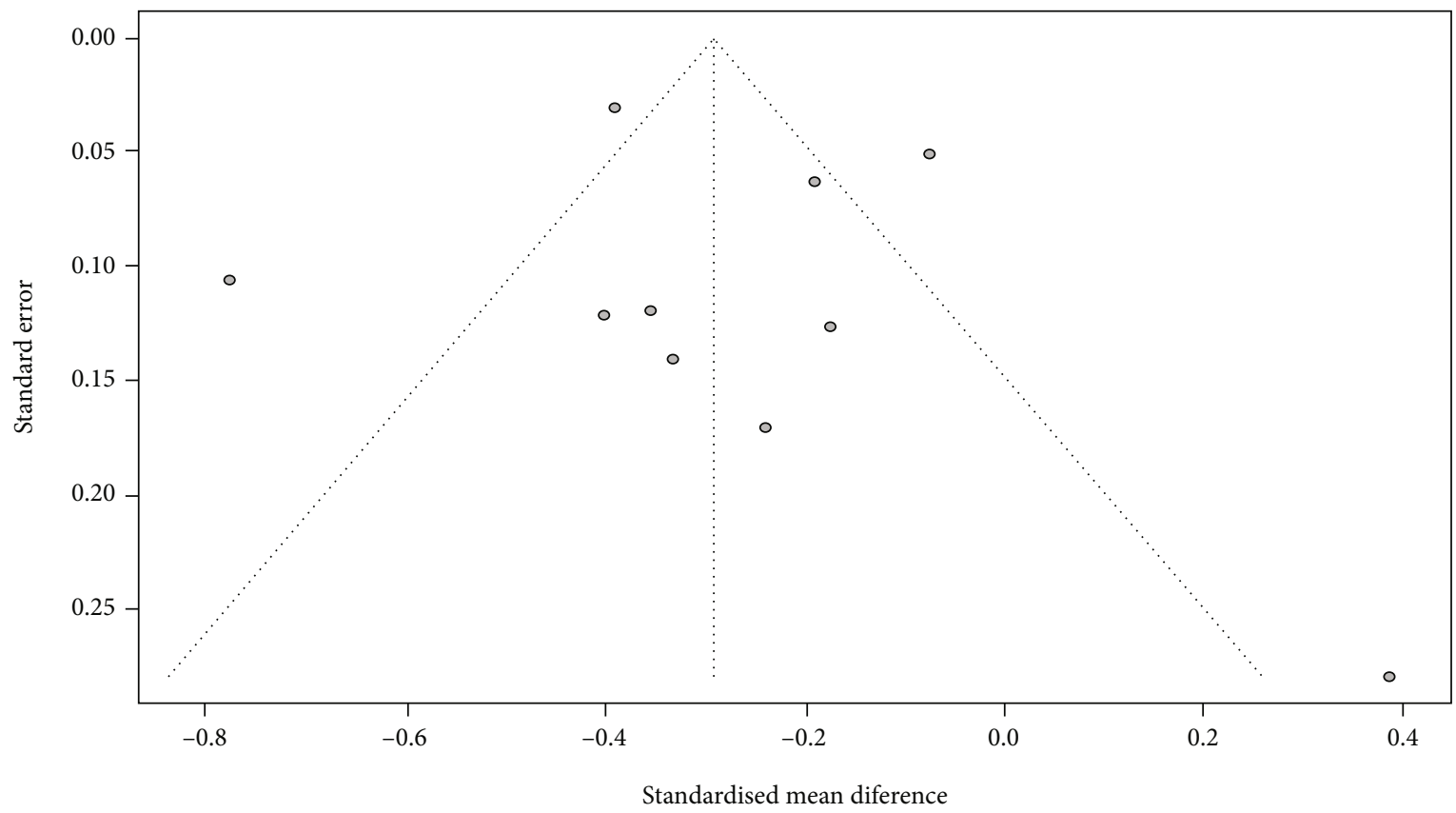

(d)

Figure 7: Funnel plots: (a) METS; (b) FBG; (c) TG; (d) HDL. 
10 studies compared HDL between patients with and without pancreatic adipose infiltration. The results presented a relatively high statistical heterogeneity $\left(I^{2}=85 \%\right.$, $p<0.01)$, and thus, a random-effects model was employed (Figure 5(a)). Each item was assumed as a potential bias factor and source of heterogeneity. Then, sensitivity analysis was conducted, and forest plots were generated (Figure 5(b)). The results exhibited high heterogeneity. Together, these results displayed that there was a trend of decreasing in HDL (SMD $=-0.29$, 95\% CI: $-0.43 \sim-0.15$ ) of adults with pancreatic adipose infiltration.

7 studies compared HTN between patients with and without pancreatic adipose infiltration. The results presented a relatively high statistical heterogeneity $\left(I^{2}=54 \%\right.$, $p=0.04)$, and thus, a random-effects model was employed (Figure 6(a)). Each item was assumed as a potential bias factor and source of heterogeneity. Then, sensitivity analysis was conducted, and forest plots were generated (Figure 6(b)). The results exhibited high heterogeneity. Taken together, there was a trend of increase in hypertension $(\mathrm{OR}=1.68$, 95\% CI: 1.32-2.13) of adults with pancreatic adipose infiltration.

3.3.3. Publication Bias. The funnel plots were basically symmetrical by visual observation, indicating no publication bias in the included studies (Figure 7).

\section{Discussion}

METS is featured as obesity, visceral fat accumulation, diabetes, hyperlipidemia, and hypertension [3, 4]. Pancreatic adipose infiltration is a component of METS similar to NAFLD. In the past, lack of appropriate survey tools that could enter the pancreas made it hard to assess pancreatic tissue in a timely and effective manner during laparoscopic or laparotomy [14]. Advanced noninvasive imaging technology makes it feasible to accurately detect and quantify pancreatic adipose $[15,16]$.

This study manifested by meta-analysis that incidence of pancreatic adipose infiltration was related to that of METS. Compared with adults without pancreatic adipose infiltration, adults with pancreatic adipose infiltration had an increasing trend of suffering from METS.

Some clinical features are bound up with METS [3], such as FBG, TG, HDL, and HTN. Meta-analysis investigated the association between them, and 11 studies were included with 17,127 participants enrolled. We found that levels of FBG $(\mathrm{SMD}=0.54,95 \% \mathrm{CI}: 0.35-0.72), \mathrm{TG} \quad(\mathrm{SMD}=0.39,95 \%$ CI: $0.25-0.53)$, and HTN (OR $=1.68,95 \% \mathrm{CI}: 1.32-2.13)$ were positively correlated with pancreatic adipose infiltration, while HDL level (SMD = -0.29, 95\% CI: $-0.43 \sim-0.15$ ) was inversely correlated with pancreatic adipose infiltration.

Choi et al. [33] unveiled that pancreatic adipose infiltration was associated with systolic hypertension rather than diastolic hypertension. As such, our results displayed a significant trend of increasing in $\mathrm{HTN}(\mathrm{OR}=1.68,95 \% \mathrm{CI}$ : $1.32-2.13$ ) in adults with pancreatic adipose infiltration. $\mathrm{Ou}$ et al. [34] illustrated that among independent risk factors such as obesity and cardiometabolic factors, pancreatic adi- pose infiltration may facilitate progression of diabetes. As detected by MRS or MRI, pancreatic fat content of subjects with T2DM is more than that of nondiabetic subjects [18, $35,36]$. Similarly, the proportion of T2DM patients in pancreatic adipose infiltration group was also higher than those in the nonpancreatic adipose infiltration group [26]. As such, we unraveled that FBG $(\mathrm{SMD}=0.54$, 95\% CI: 0.35 0.72 ) in subjects with pancreatic adipose infiltration was higher than those with nonpancreatic adipose infiltration.

Our results based on 8 studies were consistent with a meta-analysis by $\mathrm{Bi}$ et al. [13] that pancreatic adipose infiltration was associated with METS and hypertension. We analyzed 11 studies with 34,074 participants enrolled. We also analyzed correlation between pancreatic adipose infiltration and FBG, TG, and HDL, which was not been dealt with previously.

The study was limited by high heterogeneity in the results of meta-analysis and failure in identifying possible bias factors and sources of heterogeneity. It is unfortunate that during literature retrieval, no randomized controlled trials were included regarding the comparison between adults with pancreatic adipose infiltration and those without the disease. Besides, one source of weakness in this study that could have led to regional and ethnic bias was that 9 out of 11 studies were in Asia.

To sum up, pancreatic adipose infiltration presented a correlation with METS, and it may be a phenotype of METS like fatty liver and a key to treating METS. These results indicated that routine pancreatic adipose infiltration screening had clinical significance. Nevertheless, the data used in this systematic review were mainly from studies in Asia. The pathogenesis of pancreatic adipose infiltration remains unclear. Hence, more clinical trials are warranted to unravel pathophysiology, long-term complications, clinical impacts, and the formulation of therapeutic regimens.

\section{Data Availability}

The date and materials in the current study are available from the corresponding author on reasonable request.

\section{Ethical Approval}

Ethical approval is not applicable.

\section{Consent}

All authors consent to submit the manuscript for publication.

\section{Conflicts of Interest}

The authors declare no conflicts of interest.

\section{Authors' Contributions}

YZ and JL contributed to data analysis. YQX drafted and revised the article. All authors gave final approval of the version to be published and agreed to be accountable for all aspects of the work. 


\section{References}

[1] S. L. Samson and A. J. Garber, "Metabolic syndrome," Endocrinology and Metabolism Clinics of North America, vol. 43, no. 1, pp. 1-23, 2014.

[2] S. M. Grundy, "Metabolic syndrome pandemic," Arteriosclerosis, Thrombosis, and Vascular Biology, vol. 28, no. 4, pp. 629636, 2008.

[3] K. G. M. M. Alberti, P. Zimmet, and J. Shaw, "Metabolic syndrome-a new world-wide definition. A consensus statement from the International Diabetes Federation," Diabetic Medicine, vol. 23, no. 5, pp. 469-480, 2006.

[4] R. H. Eckel, S. M. Grundy, and P. Z. Zimmet, "The metabolic syndrome," Lancet, vol. 365, no. 9468, pp. 14151428, 2005.

[5] A. S. Gami, B. J. Witt, D. E. Howard et al., "Metabolic syndrome and risk of incident cardiovascular events and death: a systematic review and meta-analysis of longitudinal studies," Journal of the American College of Cardiology, vol. 49, no. 4, pp. 403-414, 2007.

[6] M. Blanquet, A. Debost-Legrand, L. Gerbaud et al., "Metabolic syndrome and social deprivation: results of a French observational multicentre survey," Family Practice, vol. 33, no. 1, pp. 17-22, 2016.

[7] A. Wree, L. Broderick, A. Canbay, H. M. Hoffman, and A. E. Feldstein, "From NAFLD to NASH to cirrhosis-new insights into disease mechanisms," Nature Reviews. Gastroenterology \& Hepatology, vol. 10, no. 11, pp. 627-636, 2013.

[8] G. Vernon, A. Baranova, and Z. M. Younossi, "Systematic review: the epidemiology and natural history of nonalcoholic fatty liver disease and non-alcoholic steatohepatitis in adults," Alimentary Pharmacology \& Therapeutics, vol. 34, no. 3, pp. 274-285, 2011.

[9] N. Chalasani, Z. Younossi, J. E. Lavine et al., "The diagnosis and management of non-alcoholic fatty liver disease: practice guideline by the American Association for the Study of Liver Diseases, American College of Gastroenterology, and the American Gastroenterological Association," Hepatology, vol. 55, no. 6, pp. 2005-2023, 2012.

[10] M. E. Rinella, "Nonalcoholic fatty liver disease: a systematic review," JAMA, vol. 313, no. 22, pp. 2263-2273, 2015.

[11] Q. M. Anstee, G. Targher, and C. P. Day, "Progression of NAFLD to diabetes mellitus, cardiovascular disease or cirrhosis," Nature Reviews. Gastroenterology \& Hepatology, vol. 10, no. 6, pp. 330-344, 2013.

[12] H. Yki-Jarvinen, "Non-alcoholic fatty liver disease as a cause and a consequence of metabolic syndrome," The Lancet Diabetes and Endocrinology, vol. 2, no. 11, pp. 901-910, 2014.

[13] Y. Bi, J. L. Wang, M. L. Li, J. Zhou, and X. L. Sun, "The association between pancreas steatosis and metabolic syndrome: a systematic review and meta-analysis," Diabetes/Metabolism Research and Reviews, vol. 35, no. 5, article e3142, 2019.

[14] J. S. Lee, S. H. Kim, D. W. Jun et al., "Clinical implications of fatty pancreas: correlations between fatty pancreas and metabolic syndrome," World Journal of Gastroenterology, vol. 15, no. 15, pp. 1869-1875, 2009.

[15] R. Catanzaro, B. Cuffari, A. Italia, and F. Marotta, "Exploring the metabolic syndrome: nonalcoholic fatty pancreas disease," World Journal of Gastroenterology, vol. 22, no. 34, pp. 76607675, 2016.
[16] M. M. Smits and E. J. M. van Geenen, "The clinical significance of pancreatic steatosis," Nature Reviews Gastroenterology \& Hepatology, vol. 8, no. 3, pp. 169-177, 2011.

[17] S. Y. Kim, H. Kim, J. Y. Cho et al., "Quantitative assessment of pancreatic fat by using unenhanced CT: pathologic correlation and clinical implications," Radiology, vol. 271, no. 1, pp. 104$112,2014$.

[18] M. E. Tushuizen, M. C. Bunck, P. J. Pouwels et al., "Pancreatic fat content and beta-cell function in men with and without type 2 diabetes," Diabetes Care, vol. 30, no. 11, pp. 29162921, 2007.

[19] H. Wang, A. Maitra, and H. Wang, "Obesity, intrapancreatic fatty infiltration, and pancreatic cancer," Clinical Cancer Research, vol. 21, no. 15, pp. 3369-3371, 2015.

[20] V. Rebours, S. Gaujoux, G. d'Assignies et al., "Obesity and fatty pancreatic infiltration are risk factors for pancreatic precancerous lesions (PanIN)," Clinical Cancer Research, vol. 21, no. 15, pp. 3522-3528, 2015.

[21] A. M. Fullenkamp, L. N. Bell, R. D. Robbins et al., "Effect of different obesogenic diets on pancreatic histology in Ossabaw miniature swine," Pancreas, vol. 40, no. 3, pp. 438443, 2011.

[22] P. S. Sepe, A. Ohri, S. Sanaka et al., "A prospective evaluation of fatty pancreas by using EUS," Gastrointestinal Endoscopy, vol. 73, no. 5, pp. 987-993, 2011.

[23] M. J. Page, J. E. McKenzie, P. M. Bossuyt et al., "The PRISMA 2020 statement: an updated guideline for reporting systematic reviews," BMJ, vol. 372, p. n71, 2021.

[24] W. C. Wu and C. Y. Wang, "Association between nonalcoholic fatty pancreatic disease (NAFPD) and the metabolic syndrome: case-control retrospective study," Cardiovascular Diabetology, vol. 12, p. 77, 2013.

[25] V. W. Wong, G. L. H. Wong, D. K. W. Yeung et al., "Fatty pancreas, insulin resistance, and $\beta$-cell function: a population study using fat-water magnetic resonance imaging," The American Journal of Gastroenterology, vol. 109, no. 4, pp. 589-597, 2014.

[26] C. Y. Wang, H. Y. Ou, M. F. Chen, T. C. Chang, and C. J. Chang, "Enigmatic ectopic fat: prevalence of nonalcoholic fatty pancreas disease and its associated factors in a Chinese population," Journal of the American Heart Association, vol. 3, no. 1, article e000297, 2014.

[27] J. Zhou, M. L. Li, D. D. Zhang et al., "The correlation between pancreatic steatosis and metabolic syndrome in a Chinese population," Pancreatology, vol. 16, no. 4, pp. 578-583, 2016.

[28] D. Wang, X. P. Yu, W. M. Xiao et al., "Prevalence and clinical characteristics of fatty pancreas in Yangzhou, China: a crosssectional study," Pancreatology, vol. 18, no. 3, pp. 263-268, 2018.

[29] H. Yamazaki, S. Tauchi, M. Kimachi et al., "Association between pancreatic fat and incidence of metabolic syndrome: a 5-year Japanese cohort study," Journal of Gastroenterology and Hepatology, vol. 33, no. 12, pp. 2048-2054, 2018.

[30] S. Weng, J. Zhou, X. Chen, Y. Sun, Z. Mao, and K. Chai, "Prevalence and factors associated with nonalcoholic fatty pancreas disease and its severity in China," Medicine (Baltimore), vol. 97, no. 26, article e11293, 2018.

[31] T. Milovanovic, S. Dragasevic, M. Stojkovic Lalosevic et al., "Ultrasonographic evaluation of fatty pancreas in Serbian patients with non alcoholic fatty liver disease-a cross sectional study," Medicina (Kaunas), vol. 55, no. 10, p. 697, 2019. 
[32] C. Chiyanika, D. F. Y. Chan, S. C. N. Hui et al., "The relationship between pancreas steatosis and the risk of metabolic syndrome and insulin resistance in Chinese adolescents with concurrent obesity and non- alcoholic fatty liver disease," Pediatric Obesity, vol. 15, no. 9, article e12653, 2020.

[33] C. W. Choi, G. H. Kim, D. H. Kang et al., "Associated factors for a hyperechogenic pancreas on endoscopic ultrasound," World Journal of Gastroenterology, vol. 16, no. 34, pp. 43294334, 2010.

[34] H. Y. Ou, C. Y. Wang, Y. C. Yang, M. F. Chen, and C. J. Chang, "The association between nonalcoholic fatty pancreas disease and diabetes," PLoS One, vol. 8, no. 5, article e62561, 2013.

[35] I. Lingvay, V. Esser, J. L. Legendre et al., "Noninvasive quantification of pancreatic fat in humans," The Journal of Clinical Endocrinology and Metabolism, vol. 94, no. 10, pp. 40704076, 2009.

[36] S. Steven, K. G. Hollingsworth, P. K. Small et al., "Weight loss decreases excess pancreatic triacylglycerol specifically in type 2 diabetes," Diabetes Care, vol. 39, no. 1, pp. 158-165, 2016. 